Themenheft Nr. 40: CoViD-19 und die digitale Hochschulbildung. Irritationen, Einsichten und Programmatiken

Herausgegeben von Markus Deimann, Marios Karapanos und Klaus Rummler

\title{
Rekonstruktion subjektiver Studienerfahrungen im (Shutdown) der Corona-Pandemie
}

\author{
Ulf-Daniel Ehlers ${ }^{1}$ (D) und Laura Eigbrecht ${ }^{1}$ (D) \\ ${ }^{1}$ Duale Hochschule Baden-Württemberg, Karlsruhe
}

\section{Zusammenfassung}

Der Beitrag berichtet über eine qualitative Studie auf Basis von seit März 2020 systematisch online durchgeführten Interviews mit Studierenden. Dabei wurden insgesamt 13 Einzel- und elf Gruppeninterviews zur persönlichen Studiensituation während des Corona-Shutdowns durchgeführt, analysiert und ausgewertet. Die Interviews geben Hinweise auf subjektive Qualitätspräferenzen und Qualitätsanforderungen an Hochschullehre, auf deren Basis sich Qualitätspotenziale für die aktuelle und zukünftige Hochschullehre formulieren lassen. Weiterhin artikulieren Studierende in den Interviews differenziert ihr Verständnis von und Anforderungen an Partizipation. Ein steigendes Bewusstsein für die Qualität von Hochschullehre scheint damit einherzugehen, diese stärker partizipativ mitgestalten zu wollen. Darüber hinaus beschreiben Studierende eigene Studienstrategien. Die aktuelle Studiensituation führt zu erhöhten Anforderungen an Selbstregulierung, Selbstlern- und Selbstorganisationskompetenzen auf individueller Ebene. Die Perspektiven der Studierenden geben Einblicke, wie sie die gemachten Erfahrungen subjektiv rekonstruieren und einordnen, welche Schlussfolgerungen sich dabei für die Hochschullehre der Zukunft ziehen lassen und wie Hochschulen sich weiterentwickeln müssen, um Studierende bestmöglich zu unterstützen. Neben zahlreichen Herausforderungen hat die Pandemie das Potenzial von Studierenden hervorgebracht, selbstbestimmt und selbstorganisiert ihre Lernprozesse zu gestalten, zu reflektieren und als Expertinnen und Experten für gute Hochschullehre zur Gestaltung des Studiums der Zukunft beizutragen.

Reconstructing subjective study experiences during shutdown in pandemic times

\begin{abstract}
The article reports on a qualitative study based on interviews with students systematically conducted online since March 2020. A total of 13 individual and eleven group interviews on the personal study situation during the Corona shutdown have been conducted, analysed and evaluated. The interviews provide information on subjective quality preferences and quality requirements for higher education, on the basis of which
\end{abstract}


quality potentials for current and future higher education teaching and learning can be formulated. Furthermore, students articulate their understanding of and requirements for participation in a differentiated manner in the interviews. A growing awareness of the quality of higher education teaching seems to go hand in hand with a greater desire to help shape it in a participatory way. Furthermore, students describe their own study strategies. The current study situation leads to increased demands for self-regulation, self-learning and self-organisation skills at the individual level. The students'perspectives provide insights into how they subjectively reconstruct and classify the experiences they have made, what conclusions can be drawn from this for future higher education and how higher education institutions must evolve in order to support students in the best possible way. In addition to numerous challenges, the pandemic has brought forth the potential of students to shape and reflect on their learning processes in a self-determined and selforganised way and to contribute as experts to shaping the future of higher education.

\section{Einleitung}

Der Corona-Shutdown hat Studierende in Hochschulen vor die Herausforderung gestellt, sich an eine örtliche Verlagerung des Studiums vom Hochschulcampus in das eigene Zuhause und eine nahezu vollständige Digitalisierung der Lehre in Form von Distance-Learning-Modi anzupassen. Dabei hat die Corona-Pandemie Hochschulen in einen der grössten bisher existierenden Feldversuche im Bereich neuer Lehr-Lernmethoden hineinversetzt (Nascimbeni und Ehlers 2020, 2). Während die Situation der Lehrenden unmittelbar nach Eintreten des Shutdowns in Form von Unterstützungsmassnahmen thematisiert wurde, geschah dies mit der Situation der Studierenden im öffentlichen Diskurs zunächst nur wenig. Grössere empirische Studien, die die Sichtweise der Studierenden fokussieren, finden sich erst circa neun Monate nach dem ersten Shutdown, gegen Ende des Jahres 2020 (Marczuk et al. 2021; Traus et al. 2020; Gestung et al. 2021). Dabei handelt es sich vor allem um quantitative, standardisierte Herangehensweisen, um die Situation der Studierenden einzuschätzen. Eine Herangehensweise im Sinne einer qualitativ-explorativen Vorgehensweise ist bislang nicht zu finden. Um die neue Studiensituation jedoch jenseits quantitativer Parameter einzuordnen, werden dazu im vorliegenden Beitrag Ergebnisse von Gesprächen mit Studierenden analysiert, die systematisch seit Beginn der Pandemie geführt wurden.

Ziel war es dabei, zu erfahren, wie Studierende das Studium während des Shutdowns hinsichtlich ihrer Fähigkeiten und hinsichtlich der vorherrschenden Rahmenbedingungen erleben, wie sie ihr Studium und Lernen selbst organisieren, und über welche Strategien der Studien- und Lerngestaltung sie verfügen beziehungsweise diese entwickeln. Dafür wurden seit März 2020 Studierende interviewt und zu ihren Studienerfahrungen während des Corona-Shutdowns befragt. Teile der Interviews 
wurden zusätzlich als Podcasts veröffentlicht und stellen die bislang umfassendste Podcastreihe zur Situation der Studierenden während der Coronazeit in ganz Europa dar (next-normal.eu und studium-im-shutdown.de). Die Daten geben Einblicke, wie Studierende die gemachten Erfahrungen subjektiv rekonstruieren.

Das Deutsche Zentrum für Hochschul- und Wissenschaftsforschung (DZHW) führte im Sommer 2020 eine standardisierte Online-Befragung namens «Studium in Zeiten der Corona-Pandemie» durch, um die Lernsituation von Studierenden und soziale Aspekte der Online-Lehre zu untersuchen (Marczuk et al. 2021). Die Studie bezieht den Bereich der individuellen Studienstrategien und Selbstorganisationsprozesse nicht mit ein. Hier ergeben sich jedoch wichtige Anschlussfragestellungen, die Auskunft geben über individuelle Lern- und Copingstrategien im Shutdown beziehungsweise den produktiv gestaltenden Umgang mit der Studiensituation. Zudem ermöglicht die pandemische Studiensituation erstmalig, Studierende zum Vergleich von Online- und Präsenzformaten zu befragen.

Dabei stehen drei Aspekte im Mittelpunkt: a) subjektive Wahrnehmung eigener Qualitätspräferenzen, b) Beteiligungs-/Handlungsmöglichkeiten und c) individuelle Studienstrategien. Die qualitative Herangehensweise eignet sich dabei besonders, subjektive Studienerfahrungen beschreiben und analysieren zu können. Der Beitrag diskutiert auch die studentische Perspektive auf die Zukunft der Hochschullehre. Dabei werden im 2. Kapitel die Forschungsmethodik und die Forschungsfragen beschrieben, um im 3. Kapitel die Ergebnisse vorzustellen und zu analysieren und anschliessend zusammenzufassen.

\section{Forschungsmethodik}

Die Studie folgt einem qualitativen Design auf Basis von online durchgeführten Interviews mit Studierenden, die anschliessend in einer Inhaltsanalyse (Mayring 2015) ausgewertet wurden. Unsere qualitative Herangehensweise fokussiert besonders auf Strategien, mit denen Studierende auf die neue Situation des Studiums im Shutdown reagieren.

\subsection{Forschungsfrage}

In der Studie geht es um die Untersuchung subjektiver Lern- und Studienpräferenzen, die Studierende hinsichtlich ihrer Studiensituation während des Shutdowns wahrnehmen und beschreiben. Die offene Hauptfragestellung lautet: Wie erleben Studierende das Studium während des Shutdowns hinsichtlich ihrer Fähigkeiten und hinsichtlich der vorherrschenden Rahmenbedingungen, um ihr Studium und Lernen selbst zu organisieren, und über welche Strategien der Studien- und Lerngestaltung verfügen beziehungsweise welche entwickeln sie? Es soll untersucht werden, ob und 
wie Studierende nicht nur im Sinne eines reaktiven «coping» (siehe beispielsweise Grabowski et al. 2007, 674 ff.), sondern im Sinne eines subjektiv produktiven Umgangs mit der neuen Studiensituation gestaltend umgehen (wie ihn beispielsweise subjektive Lern- und Sozialisationstheorien vorschlagen, siehe dazu zusammenfassend Ehlers 2004), und wie die subjektiven Rekonstruktionen Hinweise auf Studienangebote und Unterstützungsbedarfe liefern, die für die Gestaltung des Studiums im Shutdown von Bedeutung sein können. Die Hauptfragestellung konkretisiert sich in drei Unterfragen:

1. Was sind aus Studierendensicht Präferenzen und Qualitätsanforderungen an die Hochschullehre im «Shutdown??

Indem Studierende verschiedene Studienerfahrungen beschreiben, bewerten und vergleichen, geben sie Hinweise auf zugrundeliegende subjektive Qualitätspräferenzen und -anforderungen an Hochschullehre, die die Wahrnehmung von Qualität aktueller und zukünftiger Hochschullehre beeinflussen. Diese Aussagen werden hinsichtlich verschiedener Ebenen analysiert, beispielsweise hinsichtlich der digitalen Lehre und der Rahmenbedingungen dieser sowie sozialer Aspekte. Dabei wird auf die von Ehlers (2004) entwickelte Typologie subjektiver Qualitätspräferenzen zurückgegriffen.

2. Wie beschreiben Studierende ihre Partizipationsmöglichkeiten und-anforderungen in der Hochschullehre im 'Shutdown??

Partizipation im Lehr-Lernsetting unterstützt den Lernprozess. Durch Onlinelehre ändern sich die Partizipationsmöglichkeiten und neue Grenzen und Möglichkeitsspielräume werden von Lehrenden und Studierenden miteinander ausgehandelt. Dabei analysieren wir das qualitative Material anhand der Bedingungsebenen des von Mayrberger entwickelten Strukturmodells einer partizipativen Mediendidaktik (Mayrberger 2019) und des Stufenmodells von Wright (2016).

3. Wie beschreiben und rekonstruieren Studierende im Corona-〈Shutdown〉 eigene Studienstrategien, insbesondere hinsichtlich Selbstorganisation und Selbststeuerung? Die aktuelle Studiensituation führt zu erhöhten Anforderungen an Selbstregulierung, Selbstlern- und Selbstorganisationskompetenzen bei Studierenden. ${ }^{1}$ Dadurch

1 Es gibt eine Vielzahl von Definitionen und Synonymen für selbstgesteuertes Lernen, was in der Komplexität des Begriffs begründet liegt. Im deutschen Sprachgebrauch werden Begriffe wie selbstorganisiertes Lernen, autodidaktisches Lernen, selbstverantwortliches Lernen, selbstbestimmtes Lernen und selbstregulierendes Lernen verwendet. Im Angelsächsischen wird von self-directed learning, self-guided learning, self-regulated learning gesprochen. Beispielhaft hierfür steht die Definition von Konrad und Traub 1999: «Selbstgesteuertes Lernen ist eine Form des Lernens, bei der die Person in Abhängigkeit von der Art ihrer Lernmotivation selbstbestimmt eine oder mehrere Selbststeuerungsmaßnahmen (kognitiver, volitionaler, oder verhaltensmäßiger Art) ergreift und den Fortgang des Lernprozesses selbst (metakognitiv) überwacht, reguliert und bewertet» (Konrad und Traub 1999, 13). 
entsteht auch ein stärkeres Bewusstsein für die Bedeutung dieser Kompetenzen. Die Interviews werden hinsichtlich beschriebener Selbstorganisationskompetenzen und -strategien, der unterstützenden oder erschwerenden Rolle von digitalen Medien und weiteren begünstigenden oder erschwerenden Bedingungen untersucht. Dabei werden auch Ansätze des selbstgesteuerten Lernens sowie von Lernkompetenzen rekonstruiert (Ehlers 2020).

\subsection{Methodisches Design}

Das methodische Design basiert auf online geführten qualitativ vertiefenden Einzelund Gruppeninterviews. Bei der Auswertung gehen wir einerseits theoriegeleitet vor, indem wir uns in der Aufbereitung des Materials im Sinne eines deduktiven Vorgehens an Ansätzen zu subjektiven Qualitätsvorstellungen, Partizipation und Selbstorganisation orientieren (siehe Kap. 3 für die nähere Beschreibung der herangezogenen Theorien und Modelle). Andererseits sind wir am Material orientiert, indem wir bei der Materialauswertung induktiv vorgehen und das Material zur Anreicherung der Erklärungstiefe bestehender Theoriegerüste nutzen.

Die qualitativen Interviews wurden seit März 2020 als Zoom-Konferenz durchgeführt und aufgezeichnet. Dafür wurden Studierende aus verschiedenen Ländern, Studiengängen und Hochschulformen ausgewählt und in semistrukturierten, leitfadengestützten Einzel- und Gruppeninterviews von bis zu 80 Minuten Dauer zu ihrer Studiensituation im Corona-«Shutdown` befragt. Themenblöcke in den Einzelinterviews waren die vorherige im Vergleich zur aktuellen Studiensituation sowie Strategien zur Bewältigung dieser und Zukunftsperspektiven. Diese Themen wurden auch in den Gruppeninterviews besprochen, mit einer stärkeren Fokussierung auf Zukunftsaspekte und einer geweiteten Perspektive, die über die individuelle Studiensituation hinausgeht. Die digitalen Aufzeichnungen wurden über die Datenanalysesoftware MAXQDA nach der Methode der qualitativen Inhaltsanalyse (Mayring 2015) kodiert. Zuvor wurde dafür in einem theoriegestützten Verfahren ein Codebaum generiert, um damit die themenbezogene subjektive Einschätzung der Studierenden herausarbeiten zu können.

\subsection{Stichprobenauswahl und Interviewsituation}

Die Interviews wurden zwei Wochen, nachdem Hochschulen in den Modus der Onlinelehre übergehen mussten, geführt. Sie wurden partiell als Podcast aufbereitet der Öffentlichkeit zugänglich gemacht, wodurch zwei Zielsetzungen verfolgt wurden: Erkenntnisse über die Situation der Studierenden hinsichtlich der genannten Fragen zu erlangen und Studierenden in der öffentlichen Diskussion eine Stimme zu verschaffen, um ihre aktuelle Situation zu beschreiben. Diese so geschaffene 
Öffentlichkeit wurde auch von lokalen und überregionalen Medien als hoch aktuell und relevant aufgegriffen. Bis zum Zeitpunkt der Auswertung im März 2021 wurden 13 Einzelinterviews sowie elf Gruppendiskussionen durchgeführt. Dem Prinzip der maximalen Konstrastierung (Dimbath et al. 2018) entsprechend wurden dabei Studierende solcher Studiengänge ausgewählt, die hinsichtlich der von ihnen studierten Studiengänge ein diverses Fächerspektrum abdeckten (z. B. Jura, Pädagogik, Musik, Internationale Beziehungen, Medizin, Physik) und sich hinsichtlich der Hochschulbzw. Studienform unterschieden (z. B. Universitäten, Hochschulen, Duale Hochschulen, Promovierende), um den studentischen Erfahrungsraum in seiner Breite fassen zu können. Die Auswahl der Interviewpartnerinnen und Interviewpartner erfolgte einerseits über persönliche Kontakte und andererseits auch über externe Anfragen an das Interviewteam.

Die Interviewreihe umfasst Gespräche mit deutschen Studierenden sowie mit Studierenden verschiedener europäischer Länder. Zusätzlich zur Erfahrungsrealität mit dem Studium während der Pandemie wurden Anforderungen an und Visionen für ein zukünftiges Studium nach der Pandemie erfragt. Für die Veröffentlichung als Podcasts wurden die als Rohmaterial vorliegenden Interviews jeweils gekürzt, editiert und um Moderationsteile ergänzt. Sie wurden anschliessend über formatspezifische Websites und bekannte Podcast-Plattformen veröffentlicht sowie über Social Media beworben.

\subsection{Einschränkungen}

Aspekte wie Datenauswahl und Stichprobenziehung sowie die Güte der Daten schränken die Aussagekraft von empirischen Untersuchungen ein (Baur und Blasius 2014, 49, Häder 2019, 66 f.). Durch Selbstselektionseffekte wird davon ausgegangen, dass Studierende, die hinsichtlich öffentlicher Aussagen zurückhaltender sind, gar nicht erst der Teilnahme an den Interviews zustimmen könnten und eher solche zu Wort kommen, die sich wohl damit fühlen, in der Öffentlichkeit zu sprechen. Dies trifft voraussichtlich ebenso auf jene Studierenden zu, die durch ihre aktuelle Studiensituation überfordert sind. Jene und ihre speziellen Herausforderungen werden allerdings in den Interviews immer wieder angesprochen - so werden ihre Sichtweisen und Bedürfnisse zumindest teilweise eingebunden. Von einer Abbildung aller Studiensituationen und -herausforderungen kann jedoch mit diesen Einschränkungen nur sehr bedingt ausgegangen werden, was die Aussagekraft des qualitativen Materials einschränkt. 


\section{Analyse und Aufarbeitung der Ergebnisse}

\subsection{Subjektive Präferenzen und Qualitätsanforderungen an die Hochschullehre im ¿Shutdown〉}

Über das qualitative Interviewmaterial rekonstruieren wir zunächst, was aus Sicht der Lernenden Präferenzen und Qualitätsanforderungen unter Bedingungen der digitalen Hochschullehre sind. Die codierten Segmente der qualitativen Interviews wurden dabei Subcodes (Wertende Aussagen zu Formen digitaler Lehre; zu Rahmenbedingungen; Aussagen zum Thema digital social presence; Wünsche und Forderungen für die aktuelle sowie zukünftige Hochschullehre) zugeordnet und werden in diesem Kapitel hinsichtlich der Auskunft über eigene Lernpräferenzen und der subjektiven Rekonstruktion ihrer Studiensituation analysiert. Die Untersuchung subjektiver Qualitätspräferenzen wurde für das Lernen in Online-Settings erstmals durch Ehlers (2004) durchgeführt. Sie basiert auf Theoriebeständen der Selbstsozialisation (Zinnecker 2000), der subjektiven Lerntheorie nach Holzkamp (1993), des sozialökologischen Ansatzes (Bonfadelli 2001; Bronfenbrenner und Lüscher 1976; Baacke 1988; Vollbrecht 2010) und des Uses-and-Gratification-Approach (Palmgreen 1984). Die Äusserungen der Studierenden werden dabei auf die auf dem Modell subjektiver Qualität im E-Learning basierende Typologie von Anforderungspräferenzen (Qualitätstypologie) bezogen (Ehlers 2004).

In unserer Studie konnten klare Qualitätspräferenzen Studierender differenziert bewertet und daraus Qualitätspotenziale für die digitale Lehre abgeleitet werden. Diese werden in der folgenden Tabelle in Beziehung zu Aussagen von Studierenden dargestellt. Insgesamt lässt sich beobachten, dass durch die andauernde Situation mehr Rahmenbedingungen in den Blick geraten, die die wahrgenommene Qualität von Onlinelehre beeinflussen - daher steigen mit zunehmender Dauer der pandemischen Situation subjektive Qualitätsanforderungen. 
Qualitätspotenzial 1: Hochschulen begleiten Studierende durch verschiedene Services bei der Onlinelehre

- Diskrepanzerfahrung und kognitive Dissonanz: Studierende betonen, dass Hochschulen Präsenzveranstaltungen schnell digital abbilden konnten (SiS 2, 18:15; SiS 8, 05:29; SiS 6, 05:13; SiS 14, 14:52) - Studium bisher nicht als Online-Studium aufgefasst.

Qualitätspotenzial 2: Hochschulen ermöglichen Studierenden, durch verschiedene digitale Lehrformen Bedürfnissen nach Strukturierung und Flexibilität nachzukommen

- Online-Präsenzveranstaltungen helfen, den Tag zu strukturieren (SiS 10, 07:02), auch entsprechend des bekannten Tagesablaufs (SiS 1, 08:16, 11:31).

- Asynchrone Lehre durch Bereitstellung von Vorlesungsaufnahmen, Podcasts und andere Materialien erlauben eine flexiblere Tages- und Studiengestaltung (SiS 13, 07:33, 10:13) auch hinsichtlich eigener Leistungsfähigkeit je nach Tageszeit (SiS 17, 56:18).

- Wunsch nach zuverlässigen Zeiten und Terminen, wann Inhalte verfügbar sind, um sich besser strukturieren zu können (SiS 14, 13:49).

Qualitätspotenzial 3: Hochschulen erleichtern Studierenden durch individuelle Unterstützungsangebote die Professionalisierung eigener Lernprozesse

- Persönliche Verfügbarkeit und flexible Lern- und Studienunterstützung (NN 3, 22:35) sowie personalisierte Materialgestaltung (NN 2, 18:10) werden als den Lernprozess unterstützend beschrieben.

- Studierenden wird bei Klausuren oder Abgabefristen entgegengekommen, um herausfordernde Situationen aufzufangen (SiS 9, 18:11; SiS 10, 22:00) - dies wird als unterstützend beschrieben.

- Gute Erreichbarkeit von Ansprechpartnerinnen und -partnern sowie Dozierenden wird als positiv beschrieben (SiS 8, 06:18; SiS 13, 19:08) oder gefordert (SiS 3, 16:12; SiS 5, 20:41).

- Kommunikation und aufeinander Eingehen zwischen Professorinnen und Professoren und Studierenden haben sich gegenüber anderen Semestern verbessert (SiS 14, 21:34).

- Engagement von Fachschaften wird hervorgehoben, um Studierende, insbesondere im ersten Semester, zu vernetzen (SiS 14, 03:48).

- Wunsch, Dozierende hinsichtlich ihrer digitalen Lehrkompetenzen besser zu schulen (NN 1, 17:40) bzw. nach mehr Einsatz und Austausch durch Dozierende in dieser Hinsicht (SiS 5, 15:54; SiS 7, 20:34).

- Wunsch nach besserer Verfügbarkeit und Ausbau digitaler Literaturquellen (SiS 4, 09:41).

Qualitätspotenzial 4: Hochschulen schaffen Rahmenbedingungen für gleichberechtigten Zugang zum Studium auf einer geteilten Informationsbasis

- Transparente Kommunikation über Entwicklungen und Entscheidungen wird positiv bewertet (SiS 5, 02:50; SiS 7, 07:53), ihr Fehlen negativ (SiS 5, 08:56; NN 4, 22:30).

- Stark gestiegener Arbeitsumfang wird beschrieben und als belastend bewertet (SiS 14, 19:12).

- Medienkompetenzen für die erfolgreiche Teilnahme an Onlinelehre werden vorausgesetzt, zu deren Erlernen es vorher keine Angebote gegeben hat (SiS 9, 13:37).

- Nicht vorhandene oder schlechte Internetverbindung schliesst Studierende davon aus, erfolgreich am Studium teilzunehmen (NN 1, 08:30). 
Qualitätspotenzial 5: Hochschulen schaffen Raum für sozialen Austausch und innovative Ansätze für digital social presence

- Engagement von Fachschaften wird hervorgehoben, um Studierende, insbesondere im ersten Semester, zu vernetzen (SiS 14, 03:48).

- Die Möglichkeit zum sozialen Austausch in der digitalen Lehre wird als mangelhaft bewertet (SiS 5, 15:13; SiS 10, 12:08); gerade der spontane Austausch fehlt (NN 3a, 4:29) und die Möglichkeit, neue Kontakte zu knüpfen (SiS 5, 22:45).

- Sich selbst als Einzelkämpfer erleben statt Gemeinschaftsgefühl zu erfahren (SiS 2, 30:03).

- Gruppenarbeiten ermöglichen es, Freundschaften zu knüpfen (NN 9, 24:24).

- Ein Befragter ordnet den jetzigen Stand der Digitalisierung als «Digitalisierung 1.0» ein, die nicht viel Interaktion zulässt (SiS 11, 29:53).

Qualitätspotenzial 6: Hochschulen beziehen Wünsche und Bedürfnisse von Studierenden in die Gestaltung von aktueller und zukünftiger Lehre ein.

- Bedarf nach Angeboten, um eventuelle Lernrückstände vor Klausuren aufzuholen (SiS 2, 21:16).

- Digitale Angebote zumindest teilweise beibehalten, um Flexibilität zu verbessern (u. a. SiS 2, 28:21; SiS 3, 17:52; SiS 6, 27:36; SiS 8, 09:54).

- Qualität von Lehre im Blick behalten bei Entscheidung für oder gegen digitale Lehrveranstaltungen (SiS 7, 30:16).

- Hochschulen sollen Flexibilität und Änderungsbereitschaft auch nach der Pandemie beibehalten (SiS 4, 24:00, SiS 5, 10:33; SiS 10, 34:10), die zuvor bei Themen wie Nachhaltigkeit nicht vorhanden war (SiS 5, 25:24).

- Erfahrungen und Sichtweisen von Studierenden mit einbeziehen (SiS 11, 39:24) und dabei inklusive Aspekte berücksichtigen (SiS 7, 30:16).

Tab. 1.: Qualitätspotenziale (SiS = Studium im Shutdown; NN = NextNormal).

Die qualitativen Aussagen zeigen ein Bild von Studierenden, die die derzeitige Studienerfahrung vor allem hinsichtlich der Dimensionen Flexibilität, Individualisierung, Personalisierung und digital social presence rekonstruieren. Dabei gibt es Studierende, die hinsichtlich der genannten Dimensionen stärkere Herausforderungen empfinden und andere, die in Bezug auf diese Dimensionen derzeit günstige Studienbedingungen für sich vorfinden. Als besonders wichtiger Punkt kann die Entwicklung von Kapazitäten, Konzepten und Erfahrungen im Bereich der digital social presence angesehen werden. Die folgende Tabelle setzt als subjektiv wahrgenommene Qualitätsbarrieren in Beziehung zu als förderlich wahrgenommenen Bedingungen und subjektiv identifizierten Potenzialen. 


\begin{tabular}{|c|c|c|}
\hline $\begin{array}{c}\text { Subjektiv wahrgenommene } \\
\text { Qualitätsbarrieren }\end{array}$ & $\begin{array}{l}\text { Subjektiv als förderlich wahr- } \\
\text { genommene Bedingungen }\end{array}$ & $\begin{array}{c}\text { Subjektiv identifizierte } \\
\text { Potenziale }\end{array}$ \\
\hline $\begin{array}{l}\text { - Diskrepanz zwischen diffe- } \\
\text { renzierteren Qualitätsanfor- } \\
\text { derungen Studierender und } \\
\text { stattfindender Lehre }\end{array}$ & $\begin{array}{l}\text { - Präsenzvorlesungen erfül- } \\
\text { len Bedürfnis nach Struktu- } \\
\text { rierung } \\
\text { - asynchrone Formate erfül- } \\
\text { len Bedürfnis nach Flexibi- } \\
\text { lisierung }\end{array}$ & $\begin{array}{l}\text { - Reichhaltigere Lernumge- } \\
\text { bungen und ganzheitliche } \\
\text { Digitalisierung von Lehr- } \\
\text { veranstaltungen, die Quali- } \\
\text { tätsanforderungen gerecht } \\
\text { werden }\end{array}$ \\
\hline $\begin{array}{l}\text { - Belastungserfahrung durch } \\
\text { gestiegenen Arbeitsaufwand } \\
\text { - Rahmenbedingungen be- } \\
\text { einflussen Bedürfnis nach } \\
\text { gleichberechtigter Teilhabe } \\
\text { am Studium negativ }\end{array}$ & $\begin{array}{l}\text { - Bedürfnis nach Unterstüt- } \\
\text { zung wird durch organisato- } \\
\text { rische Flexibilität und Unter- } \\
\text { stützungsangebote erfüllt }\end{array}$ & $\begin{array}{l}\text { - gute Erreichbarkeit von } \\
\text { Ansprechpersonen } \\
\text { - individuelle Unterstützung } \\
\text { und Lösungen }\end{array}$ \\
\hline $\begin{array}{l}\text { - Bedürfnis nach sozialen } \\
\text { Kontakten und Austausch } \\
\text { wird nicht hinreichend } \\
\text { erfüllt }\end{array}$ & $\begin{array}{l}\text { - Bedürfnis nach sozialen } \\
\text { Kontakten wird in Einzelfäl- } \\
\text { len erfüllt, beispielsweise in } \\
\text { Gruppenarbeiten } \\
\text { - Austausch auf Augenhöhe } \\
\text { mit Dozierenden wird als } \\
\text { positiv wahrgenommen und } \\
\text { erfüllt Bedürfnisse nach } \\
\text { Respekt und Solidarität }\end{array}$ & $\begin{array}{l}\text { - neue Lösungen und Raum } \\
\text { für digital social presence } \\
\text { schaffen }\end{array}$ \\
\hline $\begin{array}{l}\text { - Sorge, dass Lehre sich ge- } \\
\text { genüber vor der Pandemie } \\
\text { nicht weiterentwickelt }\end{array}$ & $\begin{array}{l}\text { - Bedürfnis nach Qualität und } \\
\text { Weiterentwicklung von Leh- } \\
\text { re wird durch Einbezug von } \\
\text { Studierenden und Reflektie- } \\
\text { ren gemachter Erfahrungen } \\
\text { erfüllt }\end{array}$ & $\begin{array}{l}\text { - Flexibilität und Offenheit } \\
\text { beibehalten und Lehre mit } \\
\text { Studierenden weiterentwi- } \\
\text { ckeln }\end{array}$ \\
\hline
\end{tabular}

Tab. 2.: Subjektive Rekonstruktionen von Qualitätsanforderungen.

\subsection{Partizipationsmöglichkeiten und-anforderungen in der Hochschullehre im (Shutdown)}

Mayrberger beschreibt in ihrer «Partizipativen Mediendidaktik», «dass die Digitalisierung von Lehren und Lernen nur gelingen kann, wenn man den entsprechenden Akteurinnen und Akteuren ausreichend Erfahrungsräume bereitstellt, damit sie selbstgesteuertes Lernen und Partizipation erleben und authentisch erfahren - und sich dazu kritisch verhalten können» (Mayrberger 2019, 44). In ihrem Strukturmodell einer partizipativen Mediendidaktik (ebd., 189) definiert Mayrberger vier Bedingungsebenen, die nachfolgend in der Analyse reflektiert werden: die Gesellschaft (gesellschaftliche Bedingungen), den Bildungskontext (institutionelle Bedingungen), Interpersonalität (im Lehr- und Lernprozess) sowie Akteurinnen und Akteure (personaler Kontext der Lehrenden und Lernenden). Die Tiefe der Partizipation beziehungsweise Einbindung von Akteuren wird häufig anhand von Stufenmodellen eingeordnet. Ein 
grundlegendes Modell ist dabei die «Ladder of Citizen Participation» von Arnstein (1969). Ein weiteres Stufenmodell, von Wright, Block und von Unger entwickelt und insbesondere im deutschsprachigen Raum verbreitet, nennt neun Stufen der Partizipation in der Gesundheitsförderung, die auf andere Kontexte übertragen werden können: Instrumentalisierung und Anweisung (Nicht-Partizipation), Information, Anhörung und Einbeziehung (Vorstufen der Partizipation), Mitbestimmung, Teilweise Entscheidungskompetenz und Entscheidungsmacht (Partizipation) sowie Selbstorganisation, die über Partizipation hinausgeht (Wright 2016). Füchtenhans et al. (2018) beschreiben, dass studentische Mitwirkung und Mitgestaltung in Lehre und Forschung noch nicht etabliert und selbstverständlich seien, auch wenn dies eine bessere Berücksichtigung ihrer Interessen zur Folge habe und die Eigenverantwortung und Mündigkeit von Studierenden für ihren Lernprozess stärken (Füchtenhans et al. 2018, 345 f.; Hofhues 2013; Barrineau et al. 2019), um jetzige und künftige Herausforderungen der Hochschullehre angehen zu können (Mayrberger 2020). Dies entspricht auch einer zuvor bereits beschriebenen subjektiven Qualitätsperspektive, in der Lernende selbst in ihrer Verantwortung für ihren Lernprozess gestärkt werden (Ehlers 2004). Inwiefern dies in der stattfinden Onlinelehre geschieht und die Entwicklung von Selbstlern- und Selbstorganisationsstrategien beeinflussen kann, wird anhand der Aussagen in den Interviews analysiert.

Daher werden auf Grundlage des qualitativen Interviewmaterials die Partizipationsmöglichkeiten Studierender im digitalen Studium ausgewertet. In den Aussagen und Erzählfiguren der Studierenden rekonstruieren wir ihr Verständnis von Partizipation, welche Kompetenzen dafür nötig sind und welche Möglichkeiten bestehen oder gewünscht sind. Die Partizipationsformen lassen sich in aktiv-gestaltende und passive Partizipationsformen unterteilen. Darüber hinaus lassen sie sich einteilen in Partizipation auf Mikro- (Lernen, Seminar), Meso- (Hochschule) und Makroebene (Bildungsinitiativen, politische Ebene), wozu sich Beispiele in den Aussagen der Studierenden finden lassen. Zunächst wollen wir anhand der durch Studierende getroffenen Aussagen subjektiv rekonstruierte Verständnisse von Partizipation darstellen. Dabei wird die Zusammenarbeit mit Studiengangsverantwortlichen und Dozierenden genannt (SiS 17, 31:10), um die Studierendensicht bei der Erarbeitung von Lösungen einzubeziehen, auch bei der Gestaltung der Lehrveranstaltungen (SiS 13, 39:14; SiS 15, 25:10; SiS 17, 54:44; NN 9, 29:40). Hier geschieht Partizipation im Sinne der Ko-Kreation auf der Mikroebene durch Interpersonalität auf den Stufen der Einbeziehung und Mitbestimmung. Auf der Mesoebene setzt eine Universitätsleitung Empfehlungen um, die die Studierendenvertretung auf Basis einer Umfrage unter Studierenden formuliert hatte (NN 7 8, 1:02:55) und kommuniziert Entscheidungen transparent (NN 7 8, 1:01:45). Hier finden Information und Einbeziehung als Vorstufen der Partizipation statt, werden aber als Partizipationsmöglichkeit positiv bewertet. Eine Studentin erwähnt weiterhin eine Petition für die Option eines nicht 
gewerteten Semesters, welche sie als sinnvoll erachtet (SiS 9, 16:16). Hier wird Partizipation auf gesellschaftlicher Makroebene beschrieben. Es wird auch ein basaleres, passives Verständnis von Partizipation geäussert: Die Teilnahme an der Lehre an sich ist durch technische und soziale Bedingungen nicht für alle Studierenden gleichberechtigt möglich (SiS 15, 48:00; NN 3a, 33:45) - andere Personengruppen wiederum können durch die neue Flexibilität sogar besser an der Lehre teilhaben als zuvor (NN 3a, 36:00). Hier wird Partizipation strukturell und als Teilhabe verstanden und mit dem Aspekt der Chancengleichheit in Verbindung gebracht.

In den Aussagen der Studierenden finden sich auch Hinweise auf Kompetenzen und Fähigkeiten sowie Werthaltungen, die nötig sind, um Hochschulen partizipativ mitzugestalten. Diese werden einerseits bei den Studierenden selbst verortet (personale Ebene) und andererseits auf Seite der Hochschulen (Bildungskontext), um dies zu erleichtern. Auf Seiten von Studierenden wird dabei die Fähigkeit genannt, Feedback aktiv zu formulieren und so auch ohne Aufforderung Dozierende bei der Verbesserung der Qualität ihrer Lehre zu unterstützen (SiS 11, 45:09; NN 3b, 13:00). Weiterhin werden die Kenntnis von (NN 3b, 27:35), das Bewusstsein für (NN 4, 44:50, 47:00) und das Interesse und Engagement in Studierendenvertretungen beziehungsweise stärker formalisierten Partizipationskontexten genannt. Ein Student nennt die Fähigkeiten, sich auch digital zu engagieren und dadurch die überregionale Vernetzung zu verbessern (SiS 5, 27:06). Auf Seiten der Hochschulen wird eine Offenheit für Themen wie Digitalisierung sowie entsprechende Änderungsbereitschaft beobachtet, die es für gelungene Partizipation brauche und die es auch nach der Pandemie beizubehalten gelte (SiS 7, 28:08; NN 7 8; 53:28).

Schlussendlich formulieren Studierende Aussagen zu Partizipationsmöglichkeiten, die vorhanden sind oder gewünscht werden. Dabei wird insbesondere das zuvor genannte Einbeziehen der studentischen Bedürfnisse im interpersonalen Kontext der Lehrgestaltung genannt, was beibehalten und ausgebaut werden soll (SiS 7, 20:55; SiS 17, 53:31). Im Bildungskontext wird ebenso ein Einbezug der studentischen Perspektive gefordert, um die Teilhabe insbesondere jener zu unterstützen, die nicht gleichberechtigt an der digitalen Lehre partizipieren konnten (NN 9b, 1:10) sowie eine transparentere Kommunikation (SiS 15, 52:32; NN 7 8, 1:01:45), um Partizipation in passiverer Form zu begünstigen.

Inwiefern Teilhabe und Partizipation mit Werten und Bedürfnissen von Studierenden verknüpft sind, zeigen Begriffe, die Studierende bei «NextNormal» nennen, wenn sie die ideale Hochschule der Zukunft beschreiben sollen. Damit äussern Studierende Bedürfnisse, die es durch verstärkte Partizipationsmöglichkeiten zu erfüllen gilt und die überhaupt erst die Motivation zur Partizipation begründen können. In der Pandemie gibt es eine starke Betonung von Werthaltungen der Kooperation, der Solidarität und Unterstützung. Dies drückt sich aus in Werten wie «inclusive», «fair», «accessible», «student-centred» und "equal», die besonders oft von Studierenden 
artikuliert werden. Auch an anderer Stelle wird ein verstärktes Miteinander und Solidaritätsgefühl betont, das stärker wahrgenommen wird (SiS 9, 20:12). In der Diskussion um Partizipation zeigt sich auch die Verknüpfung mit Präferenzen und Qualitätsanforderungen Studierender: So wird der Wunsch gehegt, die Diskussion um Qualität von Lehre auch über die Online-Lehre hinaus prominent zu führen ( SiS 15, 49:30) und an die individuellen Bedürfnisse anzupassen (NN 4, 54:00) sowie Studierende als Expertinnen und Experten für gute Lehre in Entscheidungen mit einzubeziehen (SiS 15, 54:10).

In den qualitativen Daten zeigt sich, dass es einen stark ausgearbeiteten Korpus an Anforderungen an Partizipation gibt, der alle organisatorischen Handlungsebenen betrifft und sehr ausdifferenziert ist hinsichtlich der Partizipationsstufen und -kontexte. Sie sind verknüpft mit Wertehaltungen und entsprechenden Bedürfnissen, die Studierende mehr oder weniger bewusst durch Partizipationsmöglichkeiten artikulieren und einfordern können - sei es bei der gemeinsamen Gestaltung (digitaler) Lehre (interpersonale Aspekte), bei der Einbeziehung und Mitbestimmung bei hochschulstrategischen Entscheidungen (Bildungskontext) oder bei überregionalem und politischem Engagement. Dabei scheint ein Bewusstsein für die Qualität von Hochschullehre damit einherzugehen, diese stärker aktiv einzufordern und dabei an der Lehrgestaltung zu partizipieren. Die Aussagen zu Partizipationsmöglichkeiten und -anforderungen werden in der folgenden Tabelle zusammengefasst.

\begin{tabular}{|c|c|c|}
\hline Mikroebene & Mesoebene & Makroebene \\
\hline \multicolumn{3}{|c|}{ Aktiv-gestaltend: Jetzt } \\
\hline $\begin{array}{l}\text { - Aufforderung, Feedback } \\
\text { aktiv an Lehrende zu formu- } \\
\text { lieren } \\
\text { - gemeinsam Lehre gestalten }\end{array}$ & $\begin{array}{l}\text { - Umfrage von Studierenden- } \\
\text { vertretung mit Empfehlun- } \\
\text { gen an Hochschulleitung } \\
\text { - grössere Offenheit, wenn } \\
\text { Studierenden Themen plat- } \\
\text { zieren und voranbringen } \\
\text { wollen } \\
\text { - digitales Engagement } \\
\text { - Sichtbarkeit organisierter } \\
\text { Studierendenpartizipation } \\
\text { ist gestiegen }\end{array}$ & $\begin{array}{l}\text { - Petition für Nichtwertung } \\
\text { des Semesters }\end{array}$ \\
\hline \multicolumn{3}{|c|}{ Aktiv-gestaltend: Zukunft } \\
\hline \multicolumn{3}{|c|}{$\begin{array}{l}\text { - Offenheit soll beibehalten werden } \\
\text { - digitales Engagement überregional verstärken } \\
\text { - stärkeres organisiertes Studierendenengagement }\end{array}$} \\
\hline
\end{tabular}




\begin{tabular}{|c|c|c|}
\hline Mikroebene & Mesoebene & Makroebene \\
\hline \multicolumn{3}{|c|}{ Passiv: Jetzt } \\
\hline $\begin{array}{l}\text { - Dozierende holen Feedback } \\
\text { zu Befinden und Gestaltung } \\
\text { von Lehrveranstaltungen } \\
\text { ein } \\
\text { - Partizipation an Lehre durch } \\
\text { technische und soziale Be- } \\
\text { dingungen nicht möglich }\end{array}$ & $\begin{array}{l}\text { - Professorinnen und Profes- } \\
\text { soren kommen auf Fach- } \\
\text { schaft zu, um gemeinsam } \\
\text { Lösungen zu erarbeiten }\end{array}$ & \\
\hline \multicolumn{3}{|c|}{ Passiv: Zukunft } \\
\hline $\begin{array}{l}\text { - Dozierende sollen gezielt } \\
\text { Feedback bei Studierenden } \\
\text { einholen }\end{array}$ & $\begin{array}{l}\text { - benachteiligte Studieren- } \\
\text { dengruppen bei Gestaltung } \\
\text { zukünftiger Lehre mit ein- } \\
\text { beziehen } \\
\text { - transparente Kommunika- } \\
\text { tion }\end{array}$ & $\begin{array}{l}\text { - Hochschullehre soll inklusi- } \\
\text { ver, fairer, studierendenzen- } \\
\text { trierter und gleichberechtig- } \\
\text { ter werden }\end{array}$ \\
\hline
\end{tabular}

Tab. 3.: Aussagen zu Partizipationsmöglichkeiten und -anforderungen im Shutdown.

\subsection{Beschreibung und Rekonstruktion eigener Studienstrategien im (Shutdown)}

Anhand des qualitativen Interviewmaterials werden auch Aussagen zu Studien- und Lernstrategien der Studierenden rekonstruiert. Dabei geht es insbesondere um Strategien, mit denen Studierende ihren Studienalltag und ihren Lernprozess strukturieren und organisieren. Die Daten zeigen, dass das Studium in Zeiten der Pandemie Studierende durch räumliche und soziale Isolation stärker auf individueller Ebene fordert, Freiheitsgrade und Wahlmöglichkeiten vergrössert - und damit das Potential für die Entwicklung von Selbstorganisation und Selbstregulierung (Probst 1987, 113), mit denen sie Einfluss auf beziehungsweise Kontrolle über Handlungen und innere Prozesse nehmen (Baumeister und Vohs 2004). Weniger starke strukturierende Impulse von aussen durch Mitstudierende und räumliche Gegebenheiten sind vorgegeben und mehr Lernentscheidungen müssen durch Studierende selbst bewusst getroffen werden.

In den qualitativen Interviews machen Studierende Aussagen zu subjektiven Strategien, mit denen sie ihre Studienanforderungen bewältigen - es lässt sich schlussfolgern, dass das Bewusstsein für entsprechende Kompetenzen durch die aktuelle Situation steigt und Studierende durch die gemachten Erfahrungen ihre Kompetenzen vertiefen. In den Aussagen finden sich vielfach Selbstorganisationsbezüge. Zunächst treffen Studierende Aussagen zu Selbstorganisationsstrategien in Bezug auf Gestaltung des eigenen Lern- und Studienalltags. So sei Selbstorganisation in der aktuellen Situation besonders wichtig (SiS 10, 19:30) oder wird durch die aktuelle Situation erst erlernt (NN 9, 27:03). Eine bewusste Planung von Studien- und Freizeitphasen und Pausen ist eine von vielen Studierenden genannte Strategie (SiS 2, 23:26; SiS 5, 
19:48). Mehrere Studierende erstellen dazu Lern- bzw. Tagespläne und legen damit Zeiträume für konzentriertes Arbeiten fest (SiS 8, 08:39; SiS 10, 09:44); in manchen Fällen entspricht dies dem zuvor bekannten Studienalltag und dem Bedürfnis nach Struktur (SiS 11, 23:17). Bewusste Routinen werden als wichtig erachtet (SiS 5, 19:48) und ein Abweichen, beispielsweise durch verspätete Bereitstellung von Materialien, als problematisch (SiS 13, 14:40; SiS 14, 11:22).

Weiterhin treffen Studierende Aussagen zu Selbstorganisationsstrategien in Bezug auf Mediennutzung, welche durch die Digitalisierung der Lehre an Bedeutung gewinnen. Dabei werden ein Zuwachs an Medienkompetenzen genannt und neue digitale Tools zur Lernunterstützung genutzt (SiS 10, 16:58) wie auch digitale Lernplattformen als sinnvolle Unterstützung des selbstorganisierten Lernprozesses genannt (SiS 2, 13:40). Zahlreiche Studierende berichten von medialen Ablenkungen (SiS 4, 18:31; SiS 10, 24:48), so auch durch YouTube (SiS 5, 21:42; SiS 10, 24:25) - zudem begünstige die digitale Lehre, parallel etwas anderes zu tun und nicht mehr aufzupassen ( $\mathrm{SiS} 14,28: 11$ ) und mache auf die Mediennutzung bezogene Selbstorganisationsstrategien umso wichtiger. Es zeigt sich, dass auch hinsichtlich der Lernmotivation, der Lernfähigkeit und der Dauer der Lernepisoden Erfahrungen gemacht werden, die Studierende zunächst nicht einordnen können. Sie berichten von der verstärkten Notwendigkeit eines eigenen lernstrategischen Umgangs mit Medien, der vorher nicht auf die gleiche Weise empfunden wurde.

Darüber hinaus treffen Studierende Aussagen zu Selbstorganisationsstrategien in Bezug auf örtliche Bedingungen. Studierende nehmen ortsbezogene Aspekte der aktuellen Studiensituation sehr bewusst wahr - sie scheinen in dieser besonderen Situation bedeutsam zu sein. Die Beschränkung des Lebens- und Arbeitsraums wird von mehreren Studierenden als ungewohnt oder problematisch erachtet (SiS 2, 17:06; SiS 13, 20:07) oder sogar als herausforderndster Aspekt in der aktuellen Situation (SiS 13, 20:28). Problematische Aspekte seien Ablenkungen durch mögliche Arbeiten im Haushalt (SiS 6, 20:43; SiS 8, 15:45; SiS 11, 42:09) sowie durch weitere Personen im Haushalt (SiS 9, 25:20), wobei an anderer Stelle das Potenzial genannt wird, fokussierter und konzentrierter zu arbeiten (SiS 10, 11:21; SiS 11, 16:40). Im Umgang mit diesen Herausforderungen nennen Studierende verschiedene Strategien, um Räume bewusst zur Selbstorganisation zu nutzen. So wechselt eine Studierende im Haus ihrer Eltern die Räume, um die Bewegung auf dem Universitätscampus zu simulieren (SiS 7, 09:43). Mehrere Studierende betonen die verstärkte Wichtigkeit, es sich zu Hause «schön zu machen» (SiS 3, 13:24; SiS 4, 20:02; SiS 14, 44:53). Die Strategie, Studien- und Freizeitaktivitäten räumlich bewusst zu trennen, wird von mehreren Studierenden genannt (SiS 1, 14:28; SiS 2, 17:06; SiS 5, 09:26), beispielsweise auch durch räumliche Umstrukturierung in einer Wohngemeinschaft (SiS 5, 09:46) oder Ausweichen auf ein angemietetes Gästezimmer (SiS 9, 25:20). Ortsbezogene Aspekte können problematisch für die jeweilige Studiensituation sein, wenn beispielsweise 
ein konzentriertes Arbeiten daheim nicht möglich ist und öffentliche Lernorte wie Bibliotheken nicht verfügbar sind. Die Möglichkeiten, selbstbestimmt und -reguliert zu arbeiten, werden dadurch eingeschränkt.

Es wurde gezeigt, dass Studierende zahlreiche Selbstorganisationsstrategien kennen, reflektieren und anwenden und als bedeutsam erachten. Die Herausforderungen des digitalen Studiums liegen verstärkt auf individueller Ebene, erfordern mehr Selbstregulation und -organisation und können dadurch auch zur Belastung werden. Daher werden auch Bedingungen genannt, die die Wirksamkeit dieser Strategien einschränken. Darunter fallen zum Beispiel eine als höher wahrgenommene Arbeitsbelastung - unter anderem auch dadurch, dass digitale Formate als belastender wahrgenommen werden als Präsenzformate (SiS 7, 22:11; SiS 13, 15:53). Es wird vermutet, dass Studierende mit wenig Studienerfahrung im ersten Semester besondere Schwierigkeiten haben (SiS 14, 33:54). Weiterhin wird das Thema der mentalen Belastung mehrmals genannt - unter anderem aufgrund unsicherer Zukunftsaussichten (SiS 14, 43:30) oder indem von Freunden in psychisch herausfordernden Lebenslagen berichtet wird (SiS 6, 25:15). Das Thema <mental health sei in der aktuellen Situation herausfordernd, sie schaffe aber auch Bewusstsein und Verständnis dafür (NN $78,47: 16$ ).

Es kann daraus abgeleitet werden, dass Studieren eine noch individuellere Herausforderung geworden ist und verstärkt Selbstorganisationskompetenzen und andere Future Skills fordert und erweitert, wie beispielsweise Lernkompetenz, Selbstkompetenz, Selbstwirksamkeit (Ehlers, 2020). Die von Studierenden getroffenen Aussagen zu hinderlichen und förderlichen Faktoren hinsichtlich Selbstorganisationsstrategien fasst die folgende Tabelle zusammen:

\begin{tabular}{|c|c|c|}
\hline $\begin{array}{c}\text { Hinderliche Faktoren für die } \\
\text { Selbstorganisation }\end{array}$ & $\begin{array}{c}\text { Förderliche Faktoren für die } \\
\text { Selbstorganisation }\end{array}$ & Schlussfolgerung \\
\hline - Unplanbarkeit der Bereit- & - Bewusste Routinen und & - Bedeutung von Selbstorga- \\
stellung von Materialien & Lernpläne & nisationsstrategien steigt \\
- mediale Ablenkungen & - Zuwachs an Medienkompe- & - Notwendigkeit eines lern- \\
- Ablenkung durch Störungen & tenzen & strategischen Umgangs mit \\
und Arbeiten im Haushalt & - digitale Tools als hilfreich & Medien \\
- problematische räumliche & erachtet & - Bewusste räumliche Tren- \\
Beschränkung & - Fokussierung und Vertie- & nung Lebens- und Arbeits- \\
- höhere Arbeitsbelastung & fung zu Hause einfacher & raum \\
- psychische Belastungen & - Erfahrungen in Selbstorga- & - Räumlichkeiten umgestal- \\
- Herausforderungen auf & nisation und Selbstlernen & ten und kreativ nutzen \\
individueller Ebene & & - Unterstützungsangebote \\
\hline
\end{tabular}

Tab. 4.: Hinderliche und förderliche Faktoren für die Selbstorganisation. 


\section{Zusammenfassung und Schlussfolgerung}

Durch die besondere Studiensituation haben Studierende im Shutdown verschiedene Weisen kennengelernt, auf die Studieren möglich ist. Weiterhin haben sie neue Future Skills erworben und vertieft, die insbesondere Selbstlern- und Selbstorganisationskompetenzen umfassen und ein verstärktes Bewusstsein dafür zur Folge haben. Indem Studierende bewusster wahrnehmen, was gute Lehre für sie ausmacht, machen sie sich individuelle Qualitätsdimensionen und -anforderungen bewusst und nehmen Stärken verschiedener Lehrsettings wahr, wie beispielsweise die Flexibilität digitaler Formate und gleichzeitig die bisher nicht ausreichende Berücksichtigung von Möglichkeiten digitaler sozialer Interaktion. Weiterhin artikulieren sie, inwiefern sie bewusste Selbstorganisationsstrategien zur Gestaltung des Studienalltags nutzen, die durch Unterstützungsangebote individuell professionalisiert werden können. Durch engen Austausch mit Dozierenden konnten Studierende sich dies oft nicht nur bewusst machen, sondern eigene Präferenzen und Wünsche auch kommunizieren oder einfordern. Diese neuen partizipativen Kompetenzen können Hochschulen nutzen und von studentischen Perspektiven profitieren, um gemeinsam zukunftsfähige Lehre zu gestalten. Dies sollte als partizipativer Prozess gestaltet werden, denn das Einbeziehen studentischer Sichtweisen wird als positiv und weiteres Qualitätsmerkmal wahrgenommen. Neben zahlreichen Herausforderungen hat die Pandemie also auch das Potenzial von Studierenden hervorgebracht, selbstbestimmt und selbstorganisiert ihre Lernprozesse zu gestalten, zu reflektieren und als Expertinnen und Experten für gute Hochschullehre zur Gestaltung des Studiums der Zukunft beizutragen. Dies ist ein starkes Signal dafür, die Stimmen der Studierenden in die Debatte über die Hochschulbildung in Zeiten von CoViD-19 und in der Zukunft einzubeziehen, um eine qualitativ hochwertige, für alle zugängliche Bildung zu bieten.

\section{Literatur}

Arnstein, Sherry R. 1969. «A Ladder Of Citizen Participation». Journal of the American Institute of Planners 35 (4): 216-24. https://doi.org/10.1080/01944366908977225.

Baacke, Dieter. 1988. "Sozialökologische Ansätze in der Jugendforschung». In Handbuch der Jugendforschung, herausgegeben von Heinz-Hermann Krüger, 71-94. Wiesbaden: VS Verlag für Sozialwissenschaften. https://doi.org/10.1007/978-3-322-85702-6_5.

Barrineau, Sanna, Alexis Engström, und Ulrike Schnaas. 2019. An active student participation companion. 10.23865/hu.v9.1734. https://doi.org/10.23865/hu.v9.1734.

Baumeister, Roy F., und K. D. Vohs, Hrsg. 2004. Handbook of Self-Regulation: Research, Theory, and Applications. New York, NY: Guilford Press. http://www.loc.gov/catdir/description/guilford051/2003020013.html.

Baur, Nina, und Jörg Blasius. 2014. Handbuch Methoden der empirischen Sozialforschung. Wiesbaden: Springer Fachmedien Wiesbaden. https://doi.org/10.1007/978-3-531-18939-0. 
Bonfadelli, Heinz. 2001. Medienwirkungsforschung. 2., korrigierte Aufl. Reihe Uni-Papers 10. Konstanz: UVK-Medien.

Bronfenbrenner, Urie, und Kurt Lüscher, Hrsg. 1976. Ökologische Sozialisationsforschung. 1. Aufl. Konzepte der Humanwissenschaften. Stuttgart: Klett.

Dimbath, Oliver, Michael Ernst-Heidenreich, und Matthias Roche. 2018. «Praxis und Theorie des Theoretical Sampling. Methodologische Überlegungen zum Verfahren einer verlaufsorientierten Fallauswahl». Forum Qualitative Sozialforschung 19 (3). https://doi.org/10.17169/ FQS-19.3.2810.

Ehlers, Ulf-Daniel. 2004. Qualität im E-Learning aus Lernersicht. Wiesbaden: VS Verlag für Sozialwissenschaften. https://doi.org/10.1007/978-3-663-11211-2.

Ehlers, Ulf-Daniel. 2020. Future Skills. Wiesbaden: Springer Fachmedien Wiesbaden. https:// doi.org/10.1007/978-3-658-29297-3.

Friedrich, H. F., und Heinz Mandl. 1997. «Analyse und Förderung selbstgesteuerten Lernens». In Psychologie der Erwachsenenbildung, herausgegeben von Franz E. Weinert, und Heinz Mandl, 237-93. Enzyklopädie der Psychologie Serie I, Pädagogische Psychologie Bd. 4. Göttingen, Seattle: Hogrefe-Verlag für Pyschologie.

Füchtenhans, Stefanie, Andrea Koch-Thiele, Theresa Witt, und Katharina Zilles. 2018. «Förderung studentischer Partizipation am Beispiel einer hochschuldidaktischen Tagung». die hochschullehre 4: 345-60.

Gestung, Valeska, Ingo S. Hettler, Mandy Badermann, Ernst Deuer, und Thomas Meyer. 2021. «Online-Lehre während der COVID-19-Pandemie: Die studentische Perspektive: Forschungsbericht 7/2021». Unveröffentlichtes Manuskript. https://www.dhbw.de/fileadmin/ user_upload/Dokumente/Schrifterzeugnisse/Forschungsbericht_7_2021_Online-Lehre_ waehrend_der_COVID-19-Pandemie.pdf.

Grabowski, Joachim, Edward E. Smith, und Susan Nolen-Hoeksema, Hrsg. 2007. Atkinsons und Hilgards Einführung in die Psychologie. [2. Aufl., Übers. und Adaption der amerikan.] 14. Aufl. Heidelberg: Spektrum Akad. Verl.

Häder, Michael. 2019. Empirische Sozialforschung. Wiesbaden: Springer Fachmedien Wiesbaden. https://doi.org/10.1007/978-3-658-26986-9.

Hofhues, Sandra. 2013. "Offenheit als Stolperstein: Partizipation mit und an Hochschullehre». In Junge Hochschul- und Mediendidaktik. Forschung und Praxis im Dialog, herausgegeben von Miriam Barnat, Sandra Hofhues, Anne C. Kenneweg, Marianne Merkt, Peter Salden, und Diana Urban, 161-66. ZHW-Almanach 1. Hamburg. https://doi.org/10.3278/HSL1314W.

Holzkamp, Klaus. 1993. Lernen: Subjektwissenschaftliche Grundlegung. Frankfurt/Main: Campus-Verl.

Konrad, Klaus, und Silke Traub. 1999. Selbstgesteuertes Lernen in Theorie und Praxis. 1. Aufl. EGS-Texte. München: Oldenbourg.

Marczuk, Anna, Frank Multrus, und Markus Lörz. 2021. «Die Studiensituation in der CoronaPandemie. Auswirkungen der Digitalisierung auf die Lern- und Kontaktsituation von Studierenden». Unveröffentlichtes Manuskript. 
Martens, Samantha Eeke, Stephanie Meeuwissen, Diana Dolmans, Catherine Bovill, und Karen Könings. 2019. "Student Participation in the Design of Learning and Teaching: Disentangling the Terminology and Approaches». Medical teacher 41 (10): 1203-5. https://doi.org/10 $.1080 / 0142159 \times .2019 .1615610$.

Mayrberger, Kerstin. 2019. Partizipative Mediendidaktik: Gestaltung der (Hochschul-)Bildung unter den Bedingungen der Digitalisierung.

Mayrberger, Kerstin. 2020. «Partizipative Mediendidaktik: Darstellung von Eckpunkten und Vertiefung des Partizipationsraums als konstituierendes Strukturelement». in Jahrbuch Medienpädagogik 17, herausgegeben von Klaus Rummler, Ilka Koppel, Sandra Aßmann, Patrick Bettinger, und Karsten D. Wolf. MedienPädagogik: Zeitschrift für Theorie und Praxis der Medienbildung, 59-92. https://doi.org/10.21240/mpaed/jb17/2020.04.26.X.

Mayring, Philipp. 2015. Qualitative Inhaltsanalyse: Grundlagen und Techniken. 12., überarb. Aufl. Beltz Pädagogik. Weinheim: Beltz.

Nascimbeni, Fabio, und Ulf-Daniel Ehlers. 2020. «Open Teaching: Research and Practice on Open, Innovative and Engaging Pedagogies: Editorial». Journal of e-Learning and Knowledge Society 16 (4): I-IV. https://doi.org/10.20368/1971-8829/1135423.

Palmgreen, Philip. 1984. «Der Uses-and-Gratification-Approach: Theoretische Perspektiven und praktische Relevanz». Rundfunk und Fernsehen 32: 51-62.

Probst, Gilbert J. B. 1987. Selbst-Organisation: Ordnungsprozesse in sozialen Systemen aus ganzheitlicher Sicht. Biologie und Evolution interdisziplinär. Berlin: Parey.

Richardson, Jennifer, und Karen Swan. 2003. «Examing Social Presence in Online Courses in Relation to Students' Perceived Learning and Satisfaction». Faculty and Staff Publications and Research COLRS. http://hdl.handle.net/2142/18713.

Schmidt, Helmut, und Gerhard Stark, Hrsg. 1996. Computer Based Training in der betrieblichen Lernkultur - eine Führungsaufgabe. Sonderveröffentlichung / Bundesinstitut für Berufsbildung. Bielefeld: Bertelsmann.

Swan, Karen, und Li Fang Shih. 2019. "On the Nature and Development of Socoal Presence in Online Course Discussions». OLJ 9 (3). https://doi.org/10.24059/olj.v9i3.1788.

Traus, Anna, Katharina Höffken, Severine Thomas, Katharina Mangold, und Wolfgang Schröer. 2020. «Stu.diCo. - Studieren digital in Zeiten von Corona». Unveröffentlichtes Manuskript.

Vogel, Bernd. 2019. Orte des Selbststudiums 2018: Eine empirische Studie zur zeitlichen und räumlichen Organisation des Lernens von Studierenden. Forum Hochschulentwicklung 1/2019. Hannover: HIS-Institut für Hochschulentwicklung e.V.

Vollbrecht, Ralf. 2010. «Der sozialökologische Ansatz der Mediensozialisation». In Mediensozialisationstheorien, herausgegeben von Dagmar Hoffmann, und Lothar Mikos. 2., überarbeitete und erweiterte Auflage, 93-108. Wiesbaden: VS Verl. für Sozialwiss. https://doi. org/10.1007/978-3-531-92249-2_6.

Wright, Michael T. 2016. «Partizipation: Mitentscheidung der Bürgerinnen und Bürger». Unveröffentlichtes Manuskript.

Zinnecker, Jürgen. 2000. Selbstsozialisation. Essay über ein aktuelles Konzept: Selfsocialisation. Essay about a current theoretical debate. Weinheim: Beltz Juventa. 